\title{
Compact almost automorphic solutions for some nonlinear integral equations with time-dependent and state-dependent delay
}

\author{
El Hadi Ait Dads ${ }^{1,2}$, Fatima Boudchich ${ }^{1}$ and Brahim Es-sebbar ${ }^{1 *}$ (D)
}

"Correspondence: essebbar@live.fr 'Département de Mathématiques, Faculté des Sciences Semlalia, Université Cadi-Ayyad, BP 2390 Marrakesh, Morocco

Full list of author information is available at the end of the article

\section{每 Springer}

\begin{abstract}
We study the existence of compact almost automorphic solutions for a class of integral equations with time-dependent and state-dependent delay. An application to a blowflies model and a transmission lines model is carried out to support the theoretical finding.
\end{abstract}

Keywords: almost periodic and almost automorphic solutions; integral equations; neutral equation; state-dependent delay; Nicholson's model; lossless transmission lines

\section{Introduction}

The existence of periodic and almost periodic solutions of differential equations has an important theoretical and practical significance and is a problem of great interest. The existence of such solutions for ordinary as well as abstract differential equations has been intensively studied [1-7]. Such dynamics can be found in celestial mechanics, electronic circuits, problems of ecology and many other physical and biological systems. The parameters of such nonautonomous models are usually assumed to be periodic with respect to time due to periodic time-fluctuating environment. For example in epidemiology, the periodic aspect comes from the periodic seasonal effects. Even if the parameters of the system are periodic in time, the overall time dependence may not be periodic; i.e., if the quotient of periods of these functions is not rational, the overall time dependence will not be periodic but almost periodic in the sense of Bohr.

In reality the parameters of a system may be outputs of other almost periodic dynamical systems. However, it is well known in general that almost periodic systems do not carry necessarily almost periodic dynamics $[4,6,8]$. Although these systems may have bounded oscillating solutions, these oscillations belong to a class larger than the class of almost periodic functions, we are talking about almost automorphic functions. Bochner introduced the concept of almost automorphy in the literature in [9] as a generalization of almost periodicity. This concept was then deeply investigated by Veech [10] and many other authors. That is why it is natural to assume that the parameters of such systems are almost automorphic. Since most of such systems give rise to differential equations with solutions having bounded derivatives, a stronger concept of almost automorphy comes into play,

(c) The Author(s) 2017. This article is distributed under the terms of the Creative Commons Attribution 4.0 International License (http://creativecommons.org/licenses/by/4.0/), which permits unrestricted use, distribution, and reproduction in any medium, provided you give appropriate credit to the original author(s) and the source, provide a link to the Creative Commons license, and indicate if changes were made. 
that is, the notion of uniformly continuous almost automorphic functions. It turns out that this notion coincides with the notion of compact almost automorphy (see Lemma 6).

This paper is inspired by a work of Ding and Zou [11] where the authors investigated the existence and uniqueness of almost periodic and pseudo almost periodic solutions of the integral equation given by

$$
x(t)=\alpha(t) x(t-\sigma(t))+\int_{-\infty}^{t} \beta(t, t-s) f\left(s, x(s), x^{\prime}(s)\right) d s, \quad t \in \mathbb{R} .
$$

The authors in [11] assumed that $\sigma(t)$ is almost periodic (resp. pseudo almost periodic). In this work, we consider two variants of Eq. (1), a variant where the delay $\sigma(t)$ is compact almost automorphic in time and another variant where the delay is state-dependent. More specifically, we aim to study the existence and uniqueness of compact almost automorphic solutions for the following nonlinear time-dependent delay integral equation:

$$
x(t)=\alpha(t) x(t-\sigma(t))+\int_{-\infty}^{t} \beta(t, t-s) f\left(s, x_{s}\right) d s, \quad t \in \mathbb{R}
$$

and the following state-dependent delay one:

$$
x(t)=\alpha(t) x\left(t-\gamma\left(x_{t}\right)\right)+\int_{-\infty}^{t} \beta(t, t-s) f\left(s, x_{s}\right) d s, \quad t \in \mathbb{R},
$$

where $\alpha, \sigma, \gamma, \beta$ and $f$ are some continuous functions, and the history $x_{t}$ defined by $x_{t}(\theta):=$ $x(t+\theta)$ for each $\theta \in[-r, 0]$ belongs to the phase space $C:=C([-r, 0], \mathbb{R})$ endowed with the norm $|\varphi|_{C}:=\sup _{-r \leq \theta \leq 0}|\varphi(\theta)|$. The case where $\sigma(t)$ is almost automorphic instead of compact almost automorphic remains an open problem. In fact if the functions $\sigma(t)$ and $x(t)$ are only almost automorphic, one cannot say anything about the almost automorphy of the function $t \mapsto x(t-\sigma(t))$.

Equations similar to (2) and (3) arise in the study of heat flow in materials of fading memory type, or are in connection with epidemic problems. Motivated by a model given by Cooke and Kaplan [12], Torrejón [7] considered a nonlinear integral equation with implicit delay and investigated the existence of positive almost periodic solutions. For the same purpose, a similar class of equations was studied by Ait Dads and Ezzinbi in [13] then performed in [14] by the same authors who considered the situation where the delay is neutral and time-dependent with the following equation:

$$
x(t)-c x(t-\sigma(t))=\int_{t-r \sigma(t)}^{t} f(s, x(s)) d s, \quad t \in \mathbb{R} .
$$

Afterwards, Ait Dads et al. [2] discussed the existence of positive pseudo almost periodic solutions in the case of infinite delay for the equation

$$
x(t)=\int_{-\infty}^{t} a(t, t-s) f(s, x(s)) d s, \quad t \in \mathbb{R} .
$$

Further by 2011, Ding et al. [3] extended the above results to the following integral equation with neutral delay:

$$
x(t)=\alpha(t) x(t-\beta)+\int_{-\infty}^{t} a(t, t-s) f(s, x(s)) d s+h(t, x(t)), \quad t \in \mathbb{R} .
$$


For the case of state-dependent delay, we are inspired by the work [15] concerning the existence of bounded, periodic, and almost periodic solutions of a state-dependent delay differential equation of the form

$$
x^{\prime}(t)=F\left(t, x(t), x\left(t-\rho\left(x_{t}\right)\right)\right), \quad t \geq 0 .
$$

The paper is organized as follows. In Section 2 we recall some notations and definitions on almost periodic and almost automorphic functions. In Section 3, we give some preliminary lemmas about compact almost automorphic functions. In Section 4, we state the main results on the existence of compact almost automorphic solutions for Eq. (2) and Eq. (3). We treat also the case when the kernel in Eq. (2) is separated. In this case we prove that to obtain a compact almost automorphic solution, one only needs $f$ to be pointwise almost automorphic. At the end, in Section 5, two practical interesting examples are considered to illustrate the theoretical results.

\section{Almost periodic and almost automorphic functions}

Let $(X,\|\cdot\|)$ be a Banach space and $B C(\mathbb{R}, X)$ be the space of bounded continuous functions from $\mathbb{R}$ to $X$ equipped with the supremum norm

$$
|f|_{\infty}:=\sup _{t \in \mathbb{R}}\|f(t)\|
$$

When there is no confusion, we shall write $|f|$ instead of $|f|_{\infty}$.

Definition 1 ([16]) A continuous function $f: \mathbb{R} \rightarrow X$ is said to be Bohr almost periodic (or simply almost periodic) if, for every $\varepsilon>0$, there exists a positive number $l$ such that every interval of length $l$ contains a number $\tau$ such that

$$
\|f(t+\tau)-f(t)\|<\varepsilon \quad \text { for } t \in \mathbb{R} .
$$

Theorem 2 ([16]) Each almost periodic function is uniformly continuous.

A useful characterization of almost periodic functions was given by Bochner.

Theorem 3 ([9]) A continuous function $f: \mathbb{R} \rightarrow X$ is almost periodic if and only iffor every sequence of real numbers $\left(s_{n}\right)_{n}$ there exist a subsequence $\left(s_{n}^{\prime}\right)_{n} \subset\left(s_{n}\right)_{n}$ and a function $\tilde{f}$ such that

$$
f\left(t+s_{n}^{\prime}\right) \rightarrow \widetilde{f}(t)
$$

uniformly on $\mathbb{R}$ as $n \rightarrow \infty$.

In [17], Bochner introduced the concept of almost automorphy which is a generalization of the almost periodicity.

Definition 4 ([17]) A continuous function $f: \mathbb{R} \mapsto X$ is said to be almost automorphic if for every sequence of real numbers $\left(s_{n}\right)_{n}$ there exist a subsequence $\left(s_{n}^{\prime}\right)_{n} \subset\left(s_{n}\right)_{n}$ and a 
function $\tilde{f}$ such that for each $t \in \mathbb{R}$

$$
f\left(t+s_{n}^{\prime}\right) \rightarrow \widetilde{f}(t)
$$

and

$$
\widetilde{f}\left(t-s_{n}^{\prime}\right) \rightarrow f(t)
$$

as $n \rightarrow \infty$. If the above limits hold uniformly in compact subsets of $\mathbb{R}$, then $f$ is said to be compact almost automorphic.

Let $\mathrm{AA}(\mathbb{R}, X)$ and $\operatorname{KAA}(\mathbb{R}, X)$ denote respectively the space of almost automorphic and compact almost automorphic $X$-valued functions.

Remark By the pointwise convergence, the function $\tilde{f}$ in Definition 4 is only measurable and not necessarily continuous. If one of the two convergences in Definition 4 is uniform on $\mathbb{R}$, then $f$ becomes almost periodic. For more details about this topic, we refer the reader to the books $[18,19]$.

Definition 5 A continuous function $f: \mathbb{R} \times X \rightarrow X$ is said to be almost automorphic (resp. compact almost automorphic) in $t$ uniformly with respect to $x$ in $X$ if the following two conditions hold:

(i) for all $x \in X, f(\cdot, x) \in \mathrm{AA}(\mathbb{R}, X)$ (resp. $f(\cdot, x) \in \mathrm{KAA}(\mathbb{R}, X)$ );

(ii) $f$ is uniformly continuous on each compact set $K$ in $X$ with respect to the second variable $x$, namely, for each compact set $K$ in $X$, for all $\varepsilon>0$, there exists $\delta>0$ such that for all $x_{1}, x_{2} \in K$ one has

$$
\sup _{t \in \mathbb{R}}\left\|f\left(t, x_{1}\right)-f\left(t, x_{2}\right)\right\| \leq \varepsilon
$$

whenever $\left|x_{1}-x_{2}\right| \leq \delta$.

Denote by $\operatorname{AAU}(\mathbb{R} \times X, X)($ resp. $\operatorname{KAAU}(\mathbb{R} \times X, X))$ the set of all such functions.

\section{Some preliminary lemmas}

In this section we introduce some results concerning compact almost automorphic functions which will be used to establish the main results.

The following lemma is essential for the rest of this work. It gives a characterization of compact almost automorphic functions.

Lemma 6 ([20], Lemma 3.7) A function $f$ is compact almost automorphic if and only if it is almost automorphic and uniformly continuous.

Example Let $f: \mathbb{R} \rightarrow \mathbb{R}$ be such that

$$
f(t)=\sin \left(\frac{1}{2+\cos (t)+\cos (\sqrt{2} t)}\right)
$$


The function $f$ is almost automorphic, but it is not uniformly continuous on $\mathbb{R}$. Therefore, it is not almost periodic nor compact almost automorphic. For more details, see [21], Example 3.1.

Lemma 7 Let $y(\cdot) \in \mathrm{KAA}(\mathbb{R}, \mathbb{R})$ and $\sigma(\cdot) \in \mathrm{KAA}(\mathbb{R}, \mathbb{R})$. Then $t \mapsto y(t-\sigma(t)) \in \mathrm{KAA}(\mathbb{R}, \mathbb{R})$.

Proof Let $\left(s_{n}\right)_{n}$ be a sequence of real numbers. Then there exist a subsequence $\left(s_{n}^{\prime}\right)_{n} \subset$ $\left(s_{n}\right)_{n}$, a function $\tilde{y}: \mathbb{R} \rightarrow \mathbb{R}$, and a function $\tilde{\sigma}: \mathbb{R} \rightarrow \mathbb{R}$ such that

$$
\begin{aligned}
& y\left(t+s_{n}^{\prime}\right) \rightarrow \widetilde{y}(t), \\
& \tilde{y}\left(t-s_{n}^{\prime}\right) \rightarrow y(t), \\
& \sigma\left(t+s_{n}^{\prime}\right) \rightarrow \widetilde{\sigma}(t),
\end{aligned}
$$

and

$$
\widetilde{\sigma}\left(t-s_{n}^{\prime}\right) \rightarrow \sigma(t)
$$

as $n \rightarrow \infty$, where all the above convergences hold uniformly on compact subsets of $\mathbb{R}$. Let $I$ be a compact subset of $\mathbb{R}$. Then there exists a compact subset $\widetilde{I}$ of $\mathbb{R}$ such that, for all $t \in I$ and $n \in \mathbb{N}, t-\sigma\left(t+s_{n}^{\prime}\right) \in \widetilde{I}$ and

$$
\begin{aligned}
\left|y\left(\left(t+s_{n}^{\prime}\right)-\sigma\left(t+s_{n}^{\prime}\right)\right)-\widetilde{y}(t-\widetilde{\sigma}(t))\right| \\
\leq\left|y\left(t-\sigma\left(t+s_{n}^{\prime}\right)+s_{n}^{\prime}\right)-\widetilde{y}\left(t-\sigma\left(t+s_{n}^{\prime}\right)\right)\right| \\
\quad+\left|\widetilde{y}\left(t-\sigma\left(t+s_{n}^{\prime}\right)\right)-\widetilde{y}(t-\widetilde{\sigma}(t))\right| \\
\leq \sup _{s \in \widetilde{I}}\left|y\left(s+s_{n}^{\prime}\right)-\widetilde{y}(s)\right|+\left|\widetilde{y}\left(t-\sigma\left(t+s_{n}^{\prime}\right)\right)-\widetilde{y}(t-\widetilde{\sigma}(t))\right| \rightarrow 0
\end{aligned}
$$

as $n \rightarrow \infty$ for each $t \in I$. Using the same argument, we have

$$
\left|\widetilde{y}\left(\left(t-s_{n}^{\prime}\right)-\widetilde{\sigma}\left(t-s_{n}^{\prime}\right)\right)-y(t-\sigma(t))\right| \rightarrow 0
$$

as $n \rightarrow \infty$ for each $t \in I$. We conclude that $t \mapsto y(t-\sigma(t)) \in \mathrm{AA}(\mathbb{R}, \mathbb{R})$. We claim that $t \mapsto y(t-\sigma(t))$ is uniformly continuous. In fact, if $\left(t_{n}\right)_{n}$ and $\left(s_{n}\right)_{n}$ are two sequences such that $\left|t_{n}-s_{n}\right| \rightarrow 0$, then from the uniform continuity of $\sigma(\cdot)$ (Lemma 6) we have

$$
\left|\left(t_{n}-\sigma\left(t_{n}\right)\right)-\left(s_{n}-\sigma\left(s_{n}\right)\right)\right| \leq\left|t_{n}-s_{n}\right|+\left|\sigma\left(t_{n}\right)-\sigma\left(s_{n}\right)\right| \rightarrow 0
$$

as $n \rightarrow \infty$. Now from the uniform continuity of $y(\cdot)$ we deduce that

$$
\left|y\left(t_{n}-\sigma\left(t_{n}\right)\right)-y\left(s_{n}-\sigma\left(s_{n}\right)\right)\right| \rightarrow 0
$$

as $n \rightarrow \infty$. Therefore $t \mapsto y(t-\sigma(t))$ is uniformly continuous. It follows again by Lemma 6 that $t \mapsto y(t-\sigma(t)) \in \mathrm{KAA}(\mathbb{R}, \mathbb{R})$.

Lemma 8 Let $f$ and $g$ be both in $\operatorname{KAA}(\mathbb{R}, \mathbb{R})$, then the product $f . g$ is also in $\operatorname{KAA}(\mathbb{R}, \mathbb{R})$. 
Proof Let $\left(s_{n}\right)_{n}$ be a sequence of real numbers. Then there exist a subsequence $\left(s_{n}^{\prime}\right)_{n} \subset$ $\left(s_{n}\right)_{n}$, a function $\tilde{f}: \mathbb{R} \rightarrow \mathbb{R}$, and a function $\widetilde{g}: \mathbb{R} \rightarrow \mathbb{R}$ such that

$$
\begin{aligned}
f\left(t+s_{n}^{\prime}\right) & \rightarrow \widetilde{f}(t), \\
\widetilde{f}\left(t-s_{n}^{\prime}\right) & \rightarrow f(t), \\
g\left(t+s_{n}^{\prime}\right) & \rightarrow \widetilde{g}(t),
\end{aligned}
$$

and

$$
\tilde{g}\left(t-s_{n}^{\prime}\right) \rightarrow g(t)
$$

as $n \rightarrow \infty$, where all the above convergences hold uniformly on compact subsets of $\mathbb{R}$. Let $I$ be a compact subset of $\mathbb{R}$. Then, for $t \in I$, we have for each $t \in \mathbb{R}$

$$
\begin{aligned}
& \left|f\left(\left(t+s_{n}^{\prime}\right) g\left(t+s_{n}^{\prime}\right)\right)-\widetilde{f}(t) \widetilde{g}(t)\right| \\
& \quad \leq\left|f\left(t+s_{n}^{\prime}\right) g\left(t+s_{n}^{\prime}\right)-\widetilde{f}(t) g\left(t+s_{n}^{\prime}\right)\right|+\left|\widetilde{f}(t) g\left(t+s_{n}^{\prime}\right)-\widetilde{f}(t) \widetilde{g}(t)\right| \\
& \quad \leq|g|_{\infty}\left|f\left(t+s_{n}^{\prime}\right)-\widetilde{f}(t)\right|+|\widetilde{f}|_{\infty}\left|g\left(t+s_{n}^{\prime}\right)-\widetilde{g}(t)\right| .
\end{aligned}
$$

It follows that $f\left(t+t_{n}\right) g\left(t+t_{n}\right) \rightarrow \widetilde{f}(t) \widetilde{g}(t)$ uniformly on $I$. Similarly, we can prove that $\widetilde{f}\left(t-t_{n}\right) \widetilde{g}\left(t-t_{n}\right) \rightarrow f(t) g(t)$ uniformly on $[a, b]$. We conclude that $t \mapsto(g . f)(t)=f(t) g(t) \in$ $\operatorname{KAA}(\mathbb{R}, \mathbb{R})$.

Lemma 9 Let $f \in \operatorname{KAA}(\mathbb{R}, \mathbb{R})$ and $\beta: \mathbb{R} \times \mathbb{R}^{+} \rightarrow \mathbb{R}$ such that $t \mapsto \beta(t, \cdot) \in \operatorname{KAA}\left(\mathbb{R}, L^{1}\left(\mathbb{R}^{+}\right)\right)$. Then the function defined by

$$
F(t)=\int_{-\infty}^{t} \beta(t, t-s) f(s) d s
$$

is also in $\mathrm{KAA}(\mathbb{R}, \mathbb{R})$.

Proof Let $\left(s_{n}\right)_{n}$ be a sequence of real numbers. Then there exist a subsequence $\left(s_{n}^{\prime}\right)_{n} \subset$ $\left(s_{n}\right)_{n}$, a function $\tilde{f}: \mathbb{R} \rightarrow \mathbb{R}$, and a function $\widetilde{\beta}: \mathbb{R} \times \mathbb{R}^{+} \rightarrow \mathbb{R}$ such that

$$
\begin{aligned}
& f\left(t+s_{n}^{\prime}\right) \rightarrow \widetilde{f}(t), \\
& \widetilde{f}\left(t-s_{n}^{\prime}\right) \rightarrow f(t), \\
& \beta\left(t+s_{n}^{\prime}, \cdot\right) \rightarrow \widetilde{\beta}(t, \cdot),
\end{aligned}
$$

and

$$
\widetilde{\beta}\left(t-s_{n}^{\prime}, \cdot\right) \rightarrow \beta(t, \cdot),
$$

where all the above convergences hold uniformly for $t$ in compact subsets of $\mathbb{R}$ as $n \rightarrow \infty$. Remark that

$$
F(t)=\int_{0}^{+\infty} \beta(t, s) f(t-s) d s .
$$


Set for $t \in \mathbb{R}$ the function $\widetilde{F}$ defined by

$$
\widetilde{F}(t):=\int_{0}^{+\infty} \widetilde{\beta}(t, s) \widetilde{f}(t-s) d s
$$

Then, for each $t \in \mathbb{R}$,

$$
\begin{aligned}
& \left|F\left(t+s_{n}^{\prime}\right)-\widetilde{F}(t)\right| \\
& \leq \int_{0}^{+\infty}\left|\beta\left(t+s_{n}^{\prime}, s\right) f\left(t+s_{n}^{\prime}-s\right)-\widetilde{\beta}(t, s) \widetilde{f}(t-s)\right| d s \\
& \leq \int_{0}^{+\infty}\left|\beta\left(t+s_{n}^{\prime}, s\right) f\left(t+s_{n}^{\prime}-s\right)-\widetilde{\beta}(t, s) f\left(t+s_{n}^{\prime}-s\right)\right| d s \\
& \quad+\int_{0}^{+\infty}\left|\widetilde{\beta}(t, s) f\left(t+s_{n}^{\prime}-s\right)-\widetilde{\beta}(t, s) \widetilde{f}(t-s)\right| d s \\
& \leq|f|\left|\beta\left(t+s_{n}^{\prime}, \cdot\right)-\widetilde{\beta}(t, \cdot)\right|_{L^{1}\left(\mathbb{R}^{+}\right)}+\int_{0}^{+\infty}|\widetilde{\beta}(t, s)|\left|f\left((t-s)+s_{n}^{\prime}\right)-\widetilde{f}(t-s)\right| d s .
\end{aligned}
$$

By Lebesgue's dominated convergence theorem, we deduce that $\left|F\left(t+s_{n}^{\prime}\right)-\widetilde{F}(t)\right| \rightarrow 0$ for each $t \in \mathbb{R}$. Similarly, we can show that $\left|\widetilde{F}\left(t-s_{n}^{\prime}\right)-F(t)\right| \rightarrow 0$ for each $t \in \mathbb{R}$. Thus $F \in$ $\mathrm{AA}(\mathbb{R}, \mathbb{R})$. On the other hand, let $\left(t_{n}\right)_{n}$ and $\left(s_{n}\right)_{n}$ be two real sequences such that $\left|t_{n}-s_{n}\right| \rightarrow$ 0 as $n \rightarrow \infty$. Then, by the uniform continuity of $t \mapsto \beta(t, \cdot)$ and $f$ (Lemma 6), we have

$$
\begin{aligned}
\left|F\left(t_{n}\right)-F\left(s_{n}\right)\right| \\
\leq \int_{0}^{+\infty}\left|\beta\left(t_{n}, s\right) f\left(t_{n}-s\right)-\beta\left(s_{n}, s\right) f\left(s_{n}-s\right)\right| d s \\
\leq \int_{0}^{+\infty}\left|\beta\left(t_{n}, s\right) f\left(t_{n}-s\right)-\beta\left(s_{n}, s\right) f\left(t_{n}-s\right)\right| d s \\
\quad+\int_{0}^{+\infty}\left|\beta\left(s_{n}, s\right) f\left(t_{n}-s\right)-\beta\left(s_{n}, s\right) f\left(s_{n}-s\right)\right| d s \\
\leq \int_{0}^{+\infty}\left|\beta\left(t_{n}, s\right)-\beta\left(s_{n}, s\right)\right|\left|f\left(t_{n}-s\right)\right| d s+\int_{0}^{+\infty}\left|\beta\left(s_{n}, s\right)\right|\left|f\left(t_{n}-s\right)-f\left(s_{n}-s\right)\right| d s \\
\leq|f|\left|\beta\left(t_{n}, \cdot\right)-\beta\left(s_{n}, \cdot\right)\right|_{L^{1}\left(\mathbb{R}^{+}\right)}+\sup _{s \geq 0}\left|f\left(t_{n}-s\right)-f\left(s_{n}-s\right)\right||\beta| \rightarrow 0 \quad \text { as } n \rightarrow \infty,
\end{aligned}
$$

where $|\beta|:=\sup _{t \in \mathbb{R}}|\beta(t, \cdot)|_{L^{1}\left(\mathbb{R}^{+}\right)}$. We conclude that $F$ is uniformly continuous and thus compact almost automorphic by Lemma 6 .

The next lemma is a composition result for compact almost automorphic functions.

Lemma 10 ([22], Lemma 4.36) Let $f \in \mathrm{KAAU}(\mathbb{R} \times X, X)$ and $x \in \mathrm{KAA}(\mathbb{R}, X)$. Then $[t \mapsto$ $f(t, x(t))] \in \operatorname{KAA}(\mathbb{R}, X)$.

Lemma 11 Let $y(\cdot) \in \operatorname{KAA}(\mathbb{R}, \mathbb{R})$. Then $t \mapsto y_{t} \in \operatorname{KAA}(\mathbb{R}, C)$.

Proof Let $\left(t_{n}\right)_{n}$ be a sequence of real numbers. Then there exist a subsequence $\left(t_{n}^{\prime}\right)_{n} \subset\left(t_{n}\right)_{n}$ and a function $\tilde{y}: \mathbb{R} \rightarrow \mathbb{R}$ such that

$$
y\left(t+t_{n}\right) \rightarrow \tilde{y}(t)
$$


and

$$
\widetilde{y}\left(t-t_{n}\right) \rightarrow y(t)
$$

uniformly on compact subsets of $\mathbb{R}$ as $n \rightarrow \infty$. Let $I=[a, b]$ be a compact subset of $\mathbb{R}$. Then, for $t \in[a, b]$, we have

$$
\begin{aligned}
\left|y_{t+t_{n}}-\tilde{y}_{t}\right| & =\sup _{-r \leq \theta \leq 0}\left|y\left(t+t_{n}+\theta\right)-\tilde{y}(t+\theta)\right| \\
& =\sup _{t-r \leq u \leq t}\left|y\left(u+t_{n}\right)-\tilde{y}(u)\right| \\
& \leq \sup _{a-r \leq u \leq b}\left|y\left(u+t_{n}\right)-\tilde{y}(u)\right| .
\end{aligned}
$$

It follows that $y_{t+t_{n}} \rightarrow \tilde{y}_{t}$ uniformly on $[a, b]$. Similarly, we can prove that $\tilde{y}_{t-t_{n}} \rightarrow y_{t}$ uniformly on $[a, b]$. We conclude that $t \mapsto y_{t} \in \operatorname{KAA}(\mathbb{R}, C)$.

Remark If $y \in \mathrm{AA}(\mathbb{R}, \mathbb{R})$, then $t \mapsto y_{t}$ does not belong necessarily to $\mathrm{AA}(\mathbb{R}, C)$.

Proposition 12 ([22], Theorem 4.7) The space $\mathrm{AA}(\mathbb{R}, X)$ is a Banach space.

Corollary 13 The space $\mathrm{KAA}(\mathbb{R}, X)$ is a Banach space.

Proof Let $\left(f_{n}\right)_{n}$ be a Cauchy sequence in $\operatorname{KAA}(\mathbb{R}, X)$, then by Proposition 12 , $\left(f_{n}\right)_{n}$ converges uniformly to an almost automorphic function $f$. Since by Lemma $6 f_{n}$ is uniformly continuous for each $n \in \mathbb{N}$, then $f$ is also uniformly continuous. It follows again by Lemma 6 that $f \in \operatorname{KAA}(\mathbb{R}, X)$.

\section{Compact almost automorphic solutions of integral equations}

\subsection{Time-dependent delay integral equations}

Now consider the following integral equation:

$$
x(t)=\alpha(t) x(t-\sigma(t))+\int_{-\infty}^{t} \beta(t, t-s) f\left(s, x_{s}\right) d s .
$$

In the following, we assume that

$\left(H_{1}\right) \quad \alpha, \sigma \in \operatorname{KAA}(\mathbb{R}, \mathbb{R})$;

$\left(H_{2}\right) \quad \beta: \mathbb{R} \times \mathbb{R}^{+} \rightarrow \mathbb{R}$ satisfies $t \mapsto \beta(t, \cdot) \in \mathrm{KAA}\left(\mathbb{R}, L^{1}\left(\mathbb{R}^{+}\right)\right) ;$

$\left(H_{3}\right) f \in \mathrm{KAAU}(\mathbb{R} \times C, \mathbb{R})$. Moreover, there exists $L_{f}>0$ such that for all $t \in \mathbb{R}$ and $\phi, \psi \in C$

$$
|f(t, \phi)-f(t, \psi)| \leq L_{f}|\phi-\psi|_{C}
$$

Theorem 14 Assume that $\left(H_{1}\right)-\left(H_{3}\right)$ hold. Then Eq. (5) has a unique compact almost automorphic solution provided that

$$
|\alpha|+L_{f}|\beta|<1
$$


Proof Consider the operator $P: \operatorname{KAA}(\mathbb{R}, \mathbb{R}) \rightarrow C(\mathbb{R}, \mathbb{R})$ defined by

$$
(P x)(t):=\alpha(t) x(t-\sigma(t))+\int_{-\infty}^{t} \beta(t, t-s) f\left(s, x_{s}\right) d s \quad \text { for } t \in \mathbb{R}
$$

Using Lemmas 7, 8, 9, 10 and 11, it is clear that $P$ maps $\mathrm{KAA}(\mathbb{R}, \mathbb{R})$ into itself. For $x, y \in$ $\operatorname{KAA}(\mathbb{R}, \mathbb{R})$, we have

$$
\begin{aligned}
|P x-P y| & \leq|\alpha||x-y|+\sup _{t \in \mathbb{R}}\left(\int_{-\infty}^{t}|\beta(t, t-s)|\left|f\left(s, x_{s}\right)-f\left(s, y_{s}\right)\right| d s\right) \\
& \leq|\alpha||x-y|+L_{f} \sup _{t \in \mathbb{R}}\left(\int_{-\infty}^{t}|\beta(t, t-s)|\left|x_{s}-y_{s}\right|_{C} d s\right) \\
& \leq|\alpha||x-y|+L_{f}|x-y||\beta| \\
& \leq\left(|\alpha|+L_{f}|\beta|\right)|x-y| .
\end{aligned}
$$

Using the contraction principle on the Banach space $\operatorname{KAA}(\mathbb{R}, \mathbb{R})$, we deduce that Eq. (5) has a unique solution in $\mathrm{KAA}(\mathbb{R}, \mathbb{R})$.

To investigate the existence of non-negative compact almost automorphic solutions of Eq. (5), set the spaces

$$
\begin{aligned}
& \operatorname{KAA}^{+}(\mathbb{R}, \mathbb{R}):=\{x \in \mathrm{KAA}(\mathbb{R}, \mathbb{R}), x(t) \geq 0 \text { for all } t \in \mathbb{R}\}, \\
& C^{+}:=\{\varphi \in C, \varphi(\theta) \geq 0 \text { for all } \theta \in[-r, 0]\} .
\end{aligned}
$$

Assume that

$\left(H_{1}^{\prime}\right) \quad \alpha, \sigma \in \mathrm{KAA}^{+}(\mathbb{R}, \mathbb{R})$.

$\left(H_{2}^{\prime}\right) \beta: \mathbb{R} \times \mathbb{R}^{+} \rightarrow \mathbb{R}^{+}$satisfies $t \mapsto \beta(t, \cdot) \in \mathrm{KAA}\left(\mathbb{R}, L^{1}\left(\mathbb{R}^{+}\right)\right)$.

$\left(H_{3}^{\prime}\right) f \in \mathrm{KAAU}(\mathbb{R} \times C, \mathbb{R})$ and, for all $\varphi \in C^{+}, t \in \mathbb{R}, f(t, \varphi) \geq 0$. Moreover, there exists $L_{f}>0$ such that for all $t \in \mathbb{R}$ and $\phi, \psi \in C$

$$
|f(t, \phi)-f(t, \psi)| \leq L_{f}|\phi-\psi|_{C}
$$

Then we have the following result.

Theorem 15 Assume that $\left(H_{1}^{\prime}\right)-\left(H_{3}^{\prime}\right)$ hold. Then Eq. (5) has a unique non-negative compact almost automorphic solution provided that

$$
|\alpha|+L_{f}|\beta|<1
$$

Proof Remark just that under $\left(H_{1}^{\prime}\right)-\left(H_{3}^{\prime}\right)$ one can establish easily that Lemmas 7, 8, 9, 10 and 11 preserve non-negativity. It follows that $P$ maps $\mathrm{KAA}^{+}(\mathbb{R}, \mathbb{R})$ into itself. Moreover, as $\operatorname{KAA}^{+}(\mathbb{R}, \mathbb{R})$ is a closed subset of $\operatorname{KAA}(\mathbb{R}, \mathbb{R})$, which is a Banach space, then $\operatorname{KAA}^{+}(\mathbb{R}, \mathbb{R})$ is complete. The rest of the proof is similar to that of Theorem 14 . 


\subsection{State-dependent delay integral equations}

In what follows, we study the case where the delay depends on the history of the state; in other words, when our equation takes the form

$$
x(t)=\alpha(t) x\left(t-\gamma\left(x_{t}\right)\right)+\int_{-\infty}^{t} \beta(t, t-s) f\left(s, x_{s}\right) d s,
$$

where $\gamma: C \rightarrow \mathbb{R}^{+}$is a continuous function. We first give the following lemmas.

Lemma 16 Let $y(\cdot) \in \mathrm{KAA}(\mathbb{R}, \mathbb{R})$. If $\gamma: C \rightarrow \mathbb{R}^{+}$is uniformly continuous, then $[t \mapsto y(t-$ $\left.\left.\gamma\left(y_{t}\right)\right)\right] \in \mathrm{KAA}(\mathbb{R}, \mathbb{R})$.

Proof Since $y(\cdot) \in \operatorname{KAA}(\mathbb{R}, \mathbb{R})$, then from Lemma $11,\left[t \mapsto y_{t}\right] \in \operatorname{KAA}(\mathbb{R}, C)$. The function $\sigma: t \mapsto \gamma\left(y_{t}\right)$ is then almost automorphic and uniformly continuous. It follows by Lemma 6 that $\sigma \in \operatorname{KAA}(\mathbb{R}, \mathbb{R})$. The proof ends by applying Lemma 7 .

In what follows, we suppose that

$\left(H_{4}\right)$ (i) $\alpha, \gamma$ are Lipschitz and are in $\mathrm{KAA}(\mathbb{R}, \mathbb{R})$.

(ii) $\beta: \mathbb{R} \times \mathbb{R}^{+} \rightarrow \mathbb{R}$ satisfies $t \mapsto \beta(t, \cdot) \in \mathrm{KAA}\left(\mathbb{R}, L^{1}\left(\mathbb{R}^{+}\right)\right)$and $\beta$ is Lipschitz in the following sense:

$$
\operatorname{Lip}(\beta):=\sup _{t \neq s} \frac{|\beta(t, \cdot)-\beta(s, \cdot)|_{L^{1}\left(\mathbb{R}^{+}\right)}}{|t-s|}<\infty
$$

$\left(H_{5}\right) f \in \mathrm{KAAU}(\mathbb{R} \times C, \mathbb{R})$. Moreover, there exist $L_{f}>0, \widetilde{L}_{f}>0$ such that for all $t, s \in \mathbb{R}$ and $\phi, \psi \in C$

$$
\begin{aligned}
& |f(t, \phi)-f(t, \psi)| \leq L_{f}|\phi-\psi|_{C} . \\
& |f(t, \phi)-f(s, \phi)| \leq \widetilde{L}_{f}|t-s| .
\end{aligned}
$$

For a Lipschitz function $h$ from $(a, b)$ to $\mathbb{R}$, we define

$$
\operatorname{Lip}(h)=\sup \left\{\left|\frac{h(s)-h(t)}{t-s}\right|: s, t \in(a, b) \text { and } s \neq t\right\} .
$$

Theorem 17 Assume that $\left(H_{4}\right)$ and $\left(H_{5}\right)$ hold. Then Eq. (6) has a unique Lipschitz compact almost automorphic solution provided that

$$
\Delta:=\left(|\alpha|+L_{f}|\beta|-1\right)^{2}-4|\alpha| \operatorname{Lip}(\gamma)\left(\left(|f(\cdot, 0)|+L_{f} N\right) \operatorname{Lip}(\beta)+|\beta| \widetilde{L}_{f}+N \operatorname{Lip}(\alpha)\right)>0
$$

and

$$
|\alpha|+L_{f}|\beta|+\sqrt{\Delta}<1
$$

where $|f(\cdot, 0)|:=\sup _{t \in \mathbb{R}}|f(t, 0)|$ and $N=\frac{|f(\cdot, 0)||\beta|}{1-\left(|\alpha|+L_{f}|\beta|\right)}$ with $|f(\cdot, 0)||\beta| \neq 0$. 
Remark Condition (7) is equivalent to

$$
\operatorname{Lip}(\gamma)<\frac{\left(|\alpha|+L_{f}|\beta|-1\right)^{2}}{4|\alpha|\left(\left(|f(\cdot, 0)|+L_{f} N\right) \operatorname{Lip}(\beta)+|\beta| \widetilde{L}_{f}+N \operatorname{Lip}(\alpha)\right)},
$$

which implies that (7) is guaranteed if $\operatorname{Lip}(\gamma)$ is small enough (the effect of the delay statedependence is small).

Proof Consider the operator $P: \mathrm{KAA}(\mathbb{R}, \mathbb{R}) \rightarrow C(\mathbb{R}, \mathbb{R})$ defined by

$$
(P x)(t):=\alpha(t) x\left(t-\gamma\left(x_{t}\right)\right)+\int_{-\infty}^{t} \beta(t, t-s) f\left(s, x_{s}\right) d s \quad \text { for } t \in \mathbb{R} .
$$

Using Lemmas 9, 10 and 16 , it is clear that $P$ maps $\operatorname{KAA}(\mathbb{R}, \mathbb{R})$ into itself.

Let

$$
\left\{\begin{array}{l}
a=|\alpha| \operatorname{Lip}(\gamma) \\
b=|\alpha|+L_{f}|\beta|-1 \\
c=\left(|f(\cdot, 0)|+L_{f} N\right) \operatorname{Lip}(\beta)+|\beta| \widetilde{L}_{f}+N \operatorname{Lip}(\alpha) .
\end{array}\right.
$$

Then we have

$$
\Delta=b^{2}-4 a c .
$$

From (8) we have $b<0$, thus

$$
M:=\frac{-b+\sqrt{\Delta}}{2 a}>0
$$

and

$$
a M^{2}+b M+c=0 .
$$

Let $\Lambda$ be the subset of $\operatorname{KAA}(\mathbb{R}, X)$ defined by

$$
\Lambda:=\{x \in \operatorname{KAA}(\mathbb{R}, X): x \text { is Lipschitz, } \operatorname{Lip}(x) \leq M \text { and }|x| \leq N\} .
$$

Remark that (8) implies that

$$
|\alpha|+L_{f}|\beta|<1
$$

and thus $N \geq 0$, hence $\Lambda$ is not empty. We claim that the operator $P$ maps $\Lambda$ into itself. In fact, for $x \in \Lambda$ and $t \in \mathbb{R}$, we have

$$
\begin{aligned}
|(P x)(t)| & \leq|\alpha||x|+\left(L_{f}|x|+|f(\cdot, 0)|\right) \int_{-\infty}^{t}|\beta(t, t-s)| d s \\
& \leq|\alpha| N+\left(L_{f} N+|f(\cdot, 0)|\right) \int_{0}^{+\infty}|\beta(t, r)| d r \\
& \leq\left(|\alpha|+L_{f}|\beta|\right) N+|\beta||f(\cdot, 0)|=N .
\end{aligned}
$$


We now verify that $P x$ is Lipschitz with $\operatorname{Lip}(P x) \leq M$.

$$
\begin{aligned}
|(P x)(t)-(P x)(s)|= & \mid \alpha(t) x\left(t-\gamma\left(x_{t}\right)\right)+\int_{-\infty}^{t} \beta(t, t-\sigma) f\left(\sigma, x_{\sigma}\right) d \sigma \\
& -\alpha(s) x\left(s-\gamma\left(x_{s}\right)\right)-\int_{-\infty}^{s} \beta(s, s-\sigma) f\left(\sigma, x_{\sigma}\right) d \sigma \mid \\
\leq & \left|\alpha(t) x\left(t-\gamma\left(x_{t}\right)\right)-\alpha(s) x\left(s-\gamma\left(x_{s}\right)\right)\right| \\
& +\left|\int_{-\infty}^{t} \beta(t, t-\sigma) f\left(\sigma, x_{\sigma}\right) d \sigma-\int_{-\infty}^{s} \beta(s, s-\sigma) f\left(\sigma, x_{\sigma}\right) d \sigma\right| \\
= & I_{1}+I_{2} .
\end{aligned}
$$

On the one hand,

$$
\begin{aligned}
I_{1} & =\left|\alpha(t) x\left(t-\gamma\left(x_{t}\right)\right)-\alpha(s) x\left(s-\gamma\left(x_{s}\right)\right)\right| \\
& \leq\left|\alpha(t) x\left(t-\gamma\left(x_{t}\right)\right)-\alpha(s) x\left(t-\gamma\left(x_{t}\right)\right)\right|+\left|\alpha(s) x\left(t-\gamma\left(x_{t}\right)\right)-\alpha(s) x\left(s-\gamma\left(x_{s}\right)\right)\right| \\
& \leq|x| \operatorname{Lip}(\alpha)|t-s|+|\alpha| \operatorname{Lip}(x)\left|\left(t-\gamma\left(x_{t}\right)\right)-\left(s-\gamma\left(x_{s}\right)\right)\right| \\
& \leq|x| \operatorname{Lip}(\alpha)|t-s|+|\alpha| \operatorname{Lip}(x)\left(|t-s|+\operatorname{Lip}(\gamma)\left|x_{t}-x_{s}\right|\right) \\
& \leq|x| \operatorname{Lip}(\alpha)|t-s|+|\alpha| \operatorname{Lip}(x)(|t-s|+\operatorname{Lip}(\gamma) \operatorname{Lip}(x)|t-s|) \\
& \leq[|x| \operatorname{Lip}(\alpha)+|\alpha| \operatorname{Lip}(x)(1+\operatorname{Lip}(\gamma) \operatorname{Lip}(x))]|t-s| .
\end{aligned}
$$

On the other hand,

$$
\begin{aligned}
I_{2}= & \left|\int_{0}^{+\infty} \beta(t, \sigma) f\left(t+\sigma, x_{t+\sigma}\right) d \sigma-\int_{0}^{+\infty} \beta(s, \sigma) f\left(s+\sigma, x_{s+\sigma}\right) d \sigma\right| \\
\leq & \int_{0}^{+\infty}\left|\beta(t, \sigma) f\left(t+\sigma, x_{t+\sigma}\right)-\beta(t, \sigma) f\left(t+\sigma, x_{s+\sigma}\right)\right| d \sigma \\
& +\int_{0}^{+\infty}\left|\beta(t, \sigma) f\left(t+\sigma, x_{s+\sigma}\right)-\beta(s, \sigma) f\left(t+\sigma, x_{s+\sigma}\right)\right| d \sigma \\
& +\int_{0}^{+\infty}\left|\beta(s, \sigma) f\left(t+\sigma, x_{s+\sigma}\right)-\beta(s, \sigma) f\left(s+\sigma, x_{s+\sigma}\right)\right| d \sigma \\
\leq & \left(\sup _{t \in \mathbb{R}} \int_{0}^{+\infty}|\beta(t, \sigma)| d \sigma\right) L_{f} \operatorname{Lip}(x)|t-s|+\left(|f(t, 0)|_{\infty}+L_{f}|x|\right)\|\beta(t, \cdot)-\beta(s, \cdot)\|_{L^{1}} \\
& +|\beta| \widetilde{L}_{f}|t-s| \\
\leq & {\left[|\beta| L_{f} \operatorname{Lip}(x)+\left(|f(\cdot, 0)| L_{f}|x|\right) \operatorname{Lip}(\beta)+|\beta| \widetilde{L}_{f}\right]|t-s| . }
\end{aligned}
$$

Thus from (9) we have

$$
\begin{aligned}
& |(P x)(t)-(P x)(s)| \\
& \leq \quad\left[|x| \operatorname{Lip}(\alpha)+|\alpha| \operatorname{Lip}(x)\left(1+\operatorname{Lip}(\gamma) \operatorname{Lip}(x)+|\beta| L_{f} \operatorname{Lip}(x)\right.\right. \\
& \left.\quad+\left(|f(\cdot, 0)| L_{f}|x|\right) \operatorname{Lip}(\beta)+|\beta| \widetilde{L}_{f}\right]|t-s| \\
& \leq\left(|\alpha| \operatorname{Lip}(\gamma) M^{2}+\left(|\alpha|+|\beta| L_{f}\right) M+\left(|f(\cdot, 0)|+L_{f} N\right) \operatorname{Lip}(\beta)\right.
\end{aligned}
$$




$$
\begin{aligned}
& \left.+|\beta| \widetilde{L}_{f}+N \operatorname{Lip}(\alpha)\right)|t-s| \\
= & \left(a M^{2}+(b+1) M+c\right)|t-s| \\
= & \left(a M^{2}+b M+c+M\right)|t-s|=M|t-s| .
\end{aligned}
$$

This means that $P x \in \Lambda$. Now it suffices to prove that $P$ is a contraction on $\Lambda$. We have

$$
\begin{aligned}
|(P x)(t)-(P y)(t)| \leq & |\alpha|\left[\left|x\left(t-\gamma\left(x_{t}\right)\right)-y\left(t-\gamma\left(x_{t}\right)\right)\right|+\left|y\left(t-\gamma\left(x_{t}\right)\right)-y\left(t-\gamma\left(y_{t}\right)\right)\right|\right] \\
& +\int_{-\infty}^{t} \beta(t, t-s)\left|f\left(s, x_{s}\right)-f\left(s, y_{s}\right)\right| d s \\
\leq & |\alpha|[|x-y|+\operatorname{Lip}(y) \operatorname{Lip}(\gamma)|x-y|]+L_{f} \int_{-\infty}^{t} \beta(t, t-s)\left|x_{s}-y_{s}\right| d s \\
\leq & |\alpha|[|x-y|+\operatorname{Lip}(y) \operatorname{Lip}(\gamma)|x-y|]+L_{f}|x-y| \int_{0}^{+\infty} \beta(t, \sigma) d \sigma \\
\leq & \left(|\alpha|[1+\operatorname{Lip}(y) \operatorname{Lip}(\gamma)]+L_{f}|\beta|\right)|x-y| \\
\leq & \left(|\alpha|[1+M \operatorname{Lip}(\gamma)]+L_{f}|\beta|\right)|x-y| .
\end{aligned}
$$

Remark that

$$
|\alpha|[1+M \operatorname{Lip}(\gamma)]+L_{f}|\beta|=|\alpha|+L_{f}|\beta|+M a \leq|\alpha|+L_{f}|\beta|+\sqrt{\Delta} .
$$

Therefore, by (8), $P$ is a contraction on $\Lambda$, and thus Eq. (6) has a unique compact almost automorphic solution.

\subsection{Case of a separated kernel}

Let us consider the case where the kernel $\beta$ in Eq. (5) is separated, that is, it can be written as $\beta(t, s)=\beta_{1}(t) \beta_{2}(s)$ such that $\beta_{1} \in \mathrm{KAA}(\mathbb{R}, \mathbb{R})$ and $\beta_{2} \in L^{1}\left(\mathbb{R}^{+}, \mathbb{R}\right)$. In this special case, we will show that to obtain a compact almost automorphic solution, one only has to assume that $f \in \mathrm{AAU}(\mathbb{R} \times \mathbb{R}, \mathbb{R})$ instead of $f \in \mathrm{KAAU}(\mathbb{R} \times \mathbb{R}, \mathbb{R})$. Our equation takes the form

$$
x(t)=\alpha(t) x(t-\sigma(t))+\beta_{1}(t) \int_{-\infty}^{t} \beta_{2}(t-s) f\left(s, x_{s}\right) d s .
$$

Remark $\beta_{1} \in \operatorname{KAA}(\mathbb{R}, \mathbb{R})$ and $\beta_{2} \in L^{1}\left(\mathbb{R}^{+}, \mathbb{R}\right)$ is equivalent to the fact that $\beta: \mathbb{R} \times \mathbb{R}^{+} \rightarrow \mathbb{R}$ satisfies $t \mapsto \beta(t, \cdot) \in \mathrm{KAA}\left(\mathbb{R}, L^{1}\left(\mathbb{R}^{+}\right)\right)$.

Assume that:

$\left(H_{2}^{\prime \prime}\right) \beta: \mathbb{R} \times \mathbb{R}^{+} \rightarrow \mathbb{R}$ satisfies $\beta(t, s)=\beta_{1}(t) \beta_{2}(s)$ such that $\beta_{1} \in \mathrm{KAA}(\mathbb{R}, \mathbb{R})$ and $\beta_{2} \in$ $L^{1}\left(\mathbb{R}_{+}, \mathbb{R}\right)$.

$\left(H_{3}^{\prime \prime}\right) f \in \mathrm{AAU}(\mathbb{R} \times C, \mathbb{R})$. Moreover, there exists $L_{f}>0$ such that for all $t \in \mathbb{R}$ and $\phi, \psi \in C$

$$
|f(t, \phi)-f(t, \psi)| \leq L_{f}|\phi-\psi|_{C}
$$

Theorem 18 Let $\left(H_{1}\right),\left(H_{2}^{\prime \prime}\right)$ and $\left(H_{3}^{\prime \prime}\right)$ hold. Then Eq. (10) has a unique compact almost automorphic solution provided that

$$
|\alpha|+L_{f}|\beta|<1 .
$$


Remark Note here that the function $f$ is just in $\operatorname{AAU}(\mathbb{R} \times C, \mathbb{R})$, whereas the obtained solution $x$ is more regular, namely, $x$ is in $\operatorname{KAA}(\mathbb{R}, \mathbb{R})$.

To prove Theorem 18, we need the following lemma.

Lemma 19 Let $f \in \mathrm{AA}(\mathbb{R}, \mathbb{R})$ and $\beta: \mathbb{R} \times \mathbb{R}^{+} \rightarrow \mathbb{R}$ such that $\beta(t, s)=\beta_{1}(t) \beta_{2}(s)$, where $\beta_{1} \in \mathrm{KAA}(\mathbb{R}, \mathbb{R})$ and $\beta_{2} \in L^{1}\left(\mathbb{R}^{+}, \mathbb{R}\right)$. Then the function defined by

$$
F(t)=\int_{-\infty}^{t} \beta(t, t-s) f(s) d s
$$

is in $\mathrm{KAA}(\mathbb{R}, \mathbb{R})$.

Proof of Lemma 19 First remark that

$$
F(t)=\beta_{1}(t) \int_{-\infty}^{t} \beta_{2}(t-s) f(s) d s
$$

Since $\beta_{1} \in \operatorname{KAA}(\mathbb{R}, \mathbb{R})$, then by Lemma 8 it suffices to prove that the function defined by

$$
G(t)=\int_{-\infty}^{t} \beta_{2}(t-s) f(s) d s
$$

is in $\operatorname{KAA}(\mathbb{R}, \mathbb{R})$. We will establish that $G$ is almost automorphic, then we prove that $G$ is uniformly continuous on $\mathbb{R}$. Let $\left(t_{n}\right)_{n}$ be a sequence of real numbers. Then there exist a subsequence $\left(t_{n}^{\prime}\right)_{n} \subset\left(t_{n}\right)_{n}$ and a function $\tilde{f}: \mathbb{R} \rightarrow \mathbb{R}$ such that for each $t \in \mathbb{R}$

$$
f\left(t+t_{n}\right) \rightarrow \widetilde{f}(t)
$$

and

$$
\widetilde{f}\left(t-t_{n}\right) \rightarrow f(t)
$$

as $n \rightarrow \infty$. Let $I$ be a compact subset of $\mathbb{R}$. Set

$$
\widetilde{G}(t)=\int_{-\infty}^{t} \beta_{2}(t-s) \tilde{f}(s) d s .
$$

Then, for $t \in I$, we have

$$
\begin{aligned}
\left|G\left(t+t_{n}\right)-\tilde{G}(t)\right| & =\left|\int_{-\infty}^{t+t_{n}} \beta_{2}\left(t+t_{n}-s\right) f(s) d s-\int_{-\infty}^{t} \beta_{2}(t-s) \tilde{f}(s) d s\right| \\
& =\left|\int_{-\infty}^{t} \beta_{2}(t-s) f\left(s+t_{n}\right) d s-\int_{-\infty}^{t} \beta_{2}(t-s) \tilde{f}(s) d s\right| \\
& =\left|\int_{-\infty}^{t} \beta_{2}(t-s)\left[f\left(s+t_{n}\right)-\widetilde{f}(s)\right] d s\right| .
\end{aligned}
$$

By Lebesgue's dominated convergence theorem, the right-hand side of the above equality goes to 0 as $n$ goes to infinity. Using the same argument, we get

$$
\left|\widetilde{G}\left(t-t_{n}\right)-G(t)\right| \longrightarrow 0 \quad \text { as } n \rightarrow \infty
$$

Consequently, $G \in \mathrm{AA}(\mathbb{R}, \mathbb{R})$. 
Let $\left(t_{n}\right)_{n}$ and $\left(s_{n}\right)_{n}$ be two sequences of real numbers satisfying

$$
\left|t_{n}-s_{n}\right| \longrightarrow 0 \quad \text { as } n \rightarrow \infty
$$

We aim to show that

$$
\left|G\left(t_{n}\right)-G\left(s_{n}\right)\right| \longrightarrow 0 \quad \text { as } n \rightarrow \infty
$$

Without loss of generality, assume that $s_{n} \leq t_{n}$. We have

$$
\begin{aligned}
& \left|G\left(t_{n}\right)-G\left(s_{n}\right)\right| \\
& \quad=\left|\int_{-\infty}^{t_{n}} \beta_{2}\left(t_{n}-s\right) f(s) d s-\int_{-\infty}^{s_{n}} \beta_{2}\left(s_{n}-s\right) f(s) d s\right| \\
& \quad=\left|\int_{-\infty}^{s_{n}} \beta_{2}\left(t_{n}-s\right) f(s) d s+\int_{s_{n}}^{t_{n}} \beta_{2}\left(t_{n}-s\right) f(s) d s-\int_{-\infty}^{s_{n}} \beta_{2}\left(s_{n}-s\right) f(s) d s\right| \\
& \quad \leq\left|\int_{-\infty}^{s_{n}}\left[\beta_{2}\left(t_{n}-s\right)-\beta_{2}\left(s_{n}-s\right)\right] f(s) d s\right|+\left|\int_{s_{n}}^{t_{n}} \beta_{2}\left(t_{n}-s\right) f(s) d s\right| \\
& \quad \leq\left|\int_{0}^{+\infty}\left[\beta_{2}\left(\theta+\left(t_{n}-s_{n}\right)\right)-\beta_{2}(\theta)\right] f\left(s_{n}-\theta\right) d \theta\right|+\left|\int_{0}^{t_{n}-s_{n}} \beta_{2}(\theta) f\left(t_{n}-\theta\right) d \theta\right| \\
& \quad \leq|f|_{\infty} \int_{0}^{+\infty}\left|\beta_{2}\left(\theta+\left(t_{n}-s_{n}\right)\right)-\beta_{2}(\theta)\right| d \theta+|f|_{\infty} \int_{0}^{t_{n}-s_{n}}\left|\beta_{2}(\theta)\right| d \theta .
\end{aligned}
$$

Since $\beta_{2} \in L^{1}\left(\mathbb{R}^{+}, \mathbb{R}\right)$, then $\left|G\left(t_{n}\right)-G\left(s_{n}\right)\right| \rightarrow 0$ as $n \rightarrow \infty$. As a consequence, $G$ is uniformly continuous. By Lemma 6 we conclude that $G \in \operatorname{KAA}(\mathbb{R}, \mathbb{R})$.

Proof of Theorem 18 Consider the operator $P: \mathrm{KAA}(\mathbb{R}, \mathbb{R}) \rightarrow C(\mathbb{R}, \mathbb{R})$ defined by

$$
(P x)(t):=\alpha(t) x(t-\sigma(t))+\beta_{1}(t) \int_{-\infty}^{t} \beta_{2}(t-s) f\left(s, x_{s}\right) d s \quad \text { for } t \in \mathbb{R}
$$

Using Lemmas 7, 8, 10, 11 and 19, it is clear that $P$ maps $\mathrm{KAA}(\mathbb{R}, \mathbb{R})$ into itself. For $x, y \in$ $\operatorname{KAA}(\mathbb{R}, \mathbb{R})$, we have

$$
\begin{aligned}
|P x-P y| & \leq|\alpha||x-y|+\left|\beta_{1}\right| \int_{-\infty}^{t}\left|\beta_{2}(t-s)\right|\left|f\left(s, x_{s}\right)-f\left(s, y_{s}\right)\right| d s \\
& \leq|\alpha||x-y|+\left|\beta_{1}\right| L_{f} \int_{-\infty}^{t}\left|\beta_{2}(t-s)\right|\left|x_{s}-y_{s}\right|_{C} d s \\
& \leq|\alpha||x-y|+\left|\beta_{1}\right| L_{f}|x-y|\left|\beta_{2}\right|_{L^{1}(\mathbb{R})} \\
& \leq\left(|\alpha|+L_{f}\left|\beta_{1}\right|\left|\beta_{2}\right|_{L^{1}(\mathbb{R})} \mid\right)|x-y| .
\end{aligned}
$$

Using the contraction principle on the Banach space $\operatorname{KAA}(\mathbb{R}, \mathbb{R})$, we deduce that Eq. (10) has a unique solution in $\mathrm{KAA}(\mathbb{R}, \mathbb{R})$.

\section{Applications}

\subsection{A neutral Nicholson's blowflies model with time-dependent delay}

In the 1950s, Nicholson carried out a series of experiments to study a sheep pest, the blowfly. The flies were kept in several cages in laboratory and were observed for several 
years (see [23, 24]). After that revisited Nicholson's models appeared. Notably Gurney [25] proposed the following delayed Nicholson blowflies equation to model the population $x(t)$ of Australian sheep blowflies:

$$
\frac{d}{d t} x(t)=-\delta(t) x(t)+p(t) x(t-r) e^{-a x(t-r)} .
$$

Parameter $p$ is the maximum per capita daily egg production rate, $\frac{1}{a}$ is the size at which the blowfly population reproduces at its maximum rate, $\delta$ is the per capita daily adult death rate, and $r$ is the generation time. Recently, assuming that the biological and environmental parameters are periodic with a common period, Chen [26] considered the same equation but with periodic parameters. Now, in order to give a more generalized model, we consider a neutral equation with time-dependent delay and compact almost automorphic parameters given by

$$
\dot{x}(t)=-\delta(t) x(t)+p(t) x(t-r(t)) e^{-a(t) x(t-r(t))}+\eta(t) \dot{x}(t-r(t))+g(t) .
$$

The neutral term shall mean that not only the population $x(t)$, but also the rate $\dot{x}(t)$ has a memory effect. Assume that

(i) $a, g, \delta, \eta, p, r, \dot{r} \in \mathrm{KAA}^{+}(\mathbb{R}, \mathbb{R})$,

(ii) $\underline{\delta}=\inf _{t \in \mathbb{R}} \delta(t)>0$, inf $\operatorname{in}_{t \in \mathbb{R}}(1-\dot{r}(t))>0$, and $g(t)>0$ for all $t \in \mathbb{R}$,

(iii) the following condition holds:

$$
p(t)(1-2 a(t)) e^{-2 a(t)} \geq \delta(t) \alpha(t)+\dot{\alpha}(t)
$$

where

$$
\alpha(t)=\frac{\eta(t)}{(1-\dot{r}(t))}
$$

The previous assumptions (i) and (ii) ensure that $\alpha \in \operatorname{KAA}(\mathbb{R}, \mathbb{R})$.

We have $\eta(t)=\alpha(t)(1-\dot{r}(t))$. Replacing $\eta$ in the main Eq. (11), we get

$$
\begin{aligned}
\dot{x}(t)= & -\delta(t) x(t)+p(t) x(t-r(t)) e^{-a(t) x(t-r(t))}+\alpha(t)(1-\dot{r}(t)) \dot{x}(t-r(t))+g(t) \\
= & -\delta(t) x(t)+p(t) x(t-r(t)) e^{-a(t) x(t-r(t))}+\alpha(t) \frac{d}{d t}[x(t-r(t))]+g(t) \\
= & -\delta(t) x(t)+p(t) x(t-r(t)) e^{-a(t) x(t-r(t))}+\alpha(t) \frac{d}{d t}[x(t-r(t))] \\
& +\dot{\alpha}(t) x(t-r(t))-\dot{\alpha}(t) x(t-r(t))+g(t) \\
= & -\delta(t) x(t)+p(t) x(t-r(t)) e^{-a(t) x(t-r(t))}+\frac{d}{d t}[\alpha(t) x(t-r(t))] \\
& -\dot{\alpha}(t) x(t-r(t))+g(t) .
\end{aligned}
$$

Then

$$
\frac{d}{d t}[x(t)-\alpha(t) x(t-r(t))]=-\delta(t) x(t)+p(t) x(t-r(t)) e^{-a(t) x(t-r(t))}-\dot{\alpha}(t) x(t-r(t))+g(t) .
$$


We obtain the following neutral equation:

$$
\frac{d}{d t}[x(t)-\alpha(t) x(t-r(t))]=-\delta(t) x(t)+f\left(t, x_{t}\right)
$$

where $f(t ; \varphi):=p(t) \varphi(-r(t)) e^{-a(t) \varphi(-r(t))}-\dot{\alpha}(t) \varphi(-r(t))+g(t)$ for $\varphi \in C:=C([-r, 0] ; \mathbb{R})$. In other words,

$$
\frac{d}{d t}[x(t)-\alpha(t) x(t-r(t))]=-\delta(t)[x(t)-\alpha(t) x(t-r(t))]+F\left(t, x_{t}\right),
$$

where $F(t, \varphi):=-\delta(t) \alpha(t) \varphi(-r(t))+f(t, \varphi)$ for $\varphi \in C:=C([-r, 0] ; \mathbb{R})$.

Then, for $t \geq \sigma$,

$$
[x(t)-\alpha(t) x(t-r(t))]=e^{-\int_{\sigma}^{t} \delta(\theta) d \theta}[x(\sigma)-\alpha(\sigma) x(\sigma-r(\sigma))]+\int_{\sigma}^{t} e^{-\int_{s}^{t} \delta(\theta) d \theta} F\left(s, x_{s}\right) d s .
$$

If $x$ is a bounded solution of Eq. (14) on $\mathbb{R}$, then by letting $\sigma \rightarrow-\infty$ we obtain

$$
x(t)=\alpha(t) x(t-r(t))+\int_{-\infty}^{t} e^{-\int_{s}^{t} \delta(\theta) d \theta} F\left(s, x_{s}\right) d s .
$$

Set

$$
\beta(t, s)=e^{-\int_{t-s}^{t} \delta(\theta) d \theta} .
$$

Lemma $20 t \mapsto \beta(t, \cdot) \in \operatorname{KAA}\left(\mathbb{R}, L^{1}\left(\mathbb{R}^{+}\right)\right)$.

Proof Let $\left(t_{n}^{\prime}\right)_{n}$ be a sequence of real numbers. Then there exist a subsequence $\left(t_{n}\right)_{n} \subset\left(t_{n}^{\prime}\right)_{n}$ and a function $\tilde{\delta}: \mathbb{R} \rightarrow \mathbb{R}$ such that

$$
\delta\left(t+t_{n}\right) \rightarrow \widetilde{\delta}(t)
$$

and

$$
\widetilde{\delta}\left(t-t_{n}\right) \rightarrow \delta(t)
$$

uniformly on compact subsets of $\mathbb{R}$ as $n \rightarrow \infty$. Let $I$ be a compact subset of $\mathbb{R}$. First remark that

$$
\beta\left(t+t_{n}, s\right)=e^{-\int_{t+t_{n}-s}^{t+t_{n}} \delta(\theta) d \theta}=e^{-\int_{t-s}^{t} \delta\left(\theta+t_{n}\right) d \theta} .
$$

Let

$$
\widetilde{\beta}(t, \cdot):=e^{-\int_{t--}^{t} \widetilde{\delta}(\theta) d \theta} .
$$

Then, for $t \in I$, we have

$$
\left|\beta\left(t+t_{n}, \cdot\right)-\widetilde{\beta}(t, \cdot)\right|_{L^{1}\left(\mathbb{R}^{+}\right)}=\int_{0}^{+\infty}\left|e^{-\int_{t-s}^{t} \delta\left(\theta+t_{n}\right) d \theta}-e^{-\int_{t-\delta}^{t} \widetilde{(\theta)} d \theta}\right| d s .
$$


As the function $x \mapsto e^{-x}$ satisfies $\left|e^{-x}-e^{-y}\right| \leq|x-y|$ for $x, y \in \mathbb{R}^{+}$, we have

$$
\left|e^{-\int_{t-s}^{t} \delta\left(\theta+t_{n}\right) d \theta}-e^{-\int_{t-s}^{t} \widetilde{\delta}(\theta) d \theta}\right| \leq \int_{t-s}^{t}\left|\delta\left(\theta+t_{n}\right)-\widetilde{\delta}(\theta)\right| d \theta \rightarrow 0 \quad \text { as } n \rightarrow \infty .
$$

Since

$$
\left|e^{-\int_{t-s}^{t} \delta\left(\theta+t_{n}\right) d \theta}-e^{-\int_{t-s}^{t} \widetilde{\delta}(\theta) d \theta}\right| \leq 2 e^{-\underline{\delta s}} .
$$

It follows by Lebesgue's dominated convergence theorem that

$$
\left|\beta\left(t+t_{n}, \cdot\right)-\widetilde{\beta}(t, \cdot)\right|_{L^{1}\left(\mathbb{R}^{+}\right)} \rightarrow 0 \quad \text { for each } t \in \mathbb{R}
$$

Similarly, we can show that $\left|\widetilde{\beta}\left(t-t_{n}, \cdot\right)-\beta(t, \cdot)\right|_{L^{1}\left(\mathbb{R}^{+}\right)} \rightarrow 0$ for each $t \in \mathbb{R}$. Thus $t \mapsto \beta(t, \cdot) \in$ $\mathrm{AA}\left(\mathbb{R}, L^{1}\left(\mathbb{R}^{+}\right)\right)$. Let us prove that $t \mapsto \beta(t, \cdot)$ is uniformly continuous. In fact, let $\left(t_{n}\right)_{n}$ and $\left(s_{n}\right)_{n}$ be two real sequences such that $\left|t_{n}-s_{n}\right| \rightarrow 0$. We have

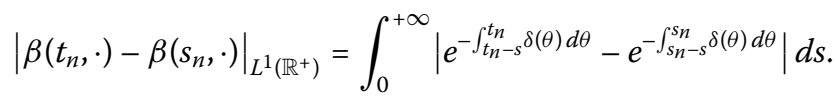

Since for each $s \geq 0$

$$
\left|e^{-\int_{t_{n}-\delta}^{t_{n}} \delta(\theta) d \theta}-e^{-\int_{n_{n}-s}^{s_{n}} \delta(\theta) d \theta}\right| \leq \int_{-s}^{0}\left|\delta\left(\theta+t_{n}\right)-\delta\left(\theta+s_{n}\right)\right| d \theta \rightarrow 0 \quad \text { as } n \rightarrow \infty,
$$

we obtain $\left|\beta\left(t_{n}, \cdot\right)-\beta\left(s_{n}, \cdot\right)\right|_{L^{1}\left(\mathbb{R}^{+}\right)} \rightarrow 0$. Consequently, $t \mapsto \beta(t, \cdot)$ is uniformly continuous and thus compact almost automorphic by Lemma 6 .

For $\varphi \in C:=C([-r, 0] ; \mathbb{R})$,

$$
\begin{aligned}
F(t, \varphi) & =-\delta(t) \alpha(t) \varphi(-r(t))+p(t) \varphi(-r(t)) e^{-a(t) \varphi(-r(t))}-\dot{\alpha}(t) \varphi(-r(t))+g(t) \\
& =\varphi(-r(t))\left[p(t) e^{-a(t) \varphi(-r(t))}-\delta(t) \alpha(t)-\dot{\alpha}(t)\right]+g(t) .
\end{aligned}
$$

Then as $g(t)>0$, for all $t \in \mathbb{R}$, and according to (12), $F(t, \varphi) \geq 0$ for all $\varphi \in C^{+}, t \in \mathbb{R}$. Moreover, $F$ is Lipschitz continuous on $C^{+}$with respect to the second variable with $\operatorname{Lip}(F)=|\delta||\alpha|+|p||a|+|\dot{\alpha}|$. As a consequence, hypotheses $\left(H_{1}^{\prime}\right)-\left(H_{3}^{\prime}\right)$ of Theorem 15 are fulfilled. Then Eq. (11) has a unique non-negative compact almost automorphic solution provided that

$$
|\alpha|+(|\delta||\alpha|+|p||a|+|\dot{\alpha}|) \frac{1}{\underline{\delta}}<1 .
$$

Remark The obtained solution is not trivial since $g(t)$ is positive.

\subsection{A lossless transmission lines model}

Actually, neutral functional differential equations are frequently used for the study of distributed networks containing lossless transmission lines. Let us consider in detail one example of this type taken from Kolmanovskii and Nosov [27]. Let the system consist of a 


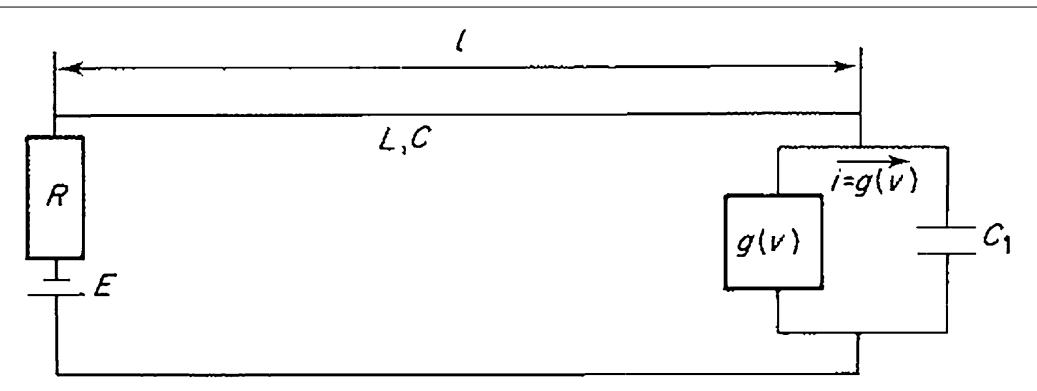

Figure 1 System with lossless transmission line [27].

long electrical line (cable) of length $l$, one end of which is switched on a power source $E$ with resistance $R_{E}$, while the other end is switched on an oscillating circuit formed by a condenser $C_{1}$ and a nonlinear element, the volt-ampere characteristic of which is $i=g(v)$ (Figure 1).

Assume that the resistance $R$ is affected by local environment conditions (principally temperature which depends on time in an oscillating way), then it can be given as a timedependent function $R(t)$ having an oscillating behavior. Let $L$ and $C$ denote the inductance and capacitance of a long line, respectively, and assume that the line is lossless.

The processes in such a system are described by the hyperbolic partial differential equations

$$
\left\{\begin{array}{l}
L \frac{\partial}{\partial t} i(x, t)=-\frac{\partial}{\partial x} v(x, t), \\
C \frac{\partial}{\partial t} v(x, t)=-\frac{\partial}{\partial x} i(x, t), \\
0<x<l, 0<t
\end{array}\right.
$$

with boundary conditions

$$
E-v(0, t)-R(t) i(0, t)=0, \quad C_{1} v_{t}(l, t)=i(l, t)-g(v(l, t)) .
$$

Let $s:=\frac{1}{\sqrt{L C}}$, and let $Z:=\sqrt{\frac{L}{C}}$ denote the wave impedance of the long line. Let

$$
K(t):=\frac{Z-R(t)}{Z+R(t)}, \quad\|K\|<1, \quad \lambda(t):=\frac{2 E}{Z+R(t)}, \quad \tau:=\frac{2 l}{s} .
$$

Designating $x(t)=v(l, t)$, then from [27] we get the following neutral differential equation:

$$
\begin{aligned}
& \frac{d}{d t}[x(t)-K(t) x(t-\tau)] \\
& \quad=C_{1} \lambda(t)-\frac{C_{1}}{Z} x(t)-\frac{C_{1} K(t)}{Z} x(t-\tau)-C_{1} g(x(t))+K(t) g(x(t-\tau)), \quad t \in \mathbb{R} .
\end{aligned}
$$

We suppose now that

(i) $g$ and $R$ are in $\operatorname{KAA}(\mathbb{R}, \mathbb{R})$,

(ii) $\underline{R}=\inf _{t \in \mathbb{R}} R(t)>0, \underline{K}=\inf _{t \in \mathbb{R}} K(t)>0$,

(iii) $g$ is Lipschitz continuous on $\mathcal{C}:=C([-\tau, 0] ; \mathbb{R})$. 
We can see that $x$ is a bounded solution of Eq. (18) if and only if it satisfies

$$
x(t)=K(t) x(t-\tau)+\int_{-\infty}^{t} e^{-\frac{C_{1}}{Z}(t-s)} f\left(s, x_{s}\right) d s
$$

with

$$
f(t, \varphi):=-2 \frac{C_{1}}{Z} K(t) \varphi(-\tau)+C_{1} \lambda(t)-C_{1} g(\varphi(0))-C_{1} K(t) g(\varphi(-\tau)), \quad \varphi \in \mathcal{C} .
$$

We have

$$
\begin{aligned}
|f(t, \varphi)-f(t, \psi)| & \leq\left(\frac{2}{Z}+\operatorname{Lip}(g)\right)|K| C_{1}|\varphi(-\tau)-\psi(-\tau)|+C_{1} \operatorname{Lip}(g)|\varphi(0)-\psi(0)| \\
& \leq\left(\frac{2}{Z}|K|+\operatorname{Lip}(g)(1+|K|)\right) C_{1}|\varphi-\psi|_{\mathcal{C}} .
\end{aligned}
$$

From (i)-(iii) we get that $K(t) \in \mathrm{KAA}(\mathbb{R}), f \in \mathrm{KAAU}(\mathbb{R} \times \mathcal{C}, \mathbb{R})$ and $f$ is Lipschitz continuous with $\operatorname{Lip}(f)=\left(\frac{2}{Z}|K|+\operatorname{Lip}(g)(1+|K|)\right) C_{1}$. Then hypotheses $\left(H_{1}\right)-\left(H_{3}\right)$ of Theorem 14 are satisfied. Consequently, Eq. (18) admits a unique compact almost automorphic solution provided that

$$
|K|+Z\left(\frac{2}{Z}|K|+\operatorname{Lip}(g)(1+|K|)\right)<1 .
$$

\section{Competing interests}

The authors declare that they have no competing interests.

\section{Authors' contributions}

All authors read and approved the final manuscript.

\section{Author details}

'Département de Mathématiques, Faculté des Sciences Semlalia, Université Cadi-Ayyad, BP 2390, Marrakesh, Morocco.

${ }^{2}$ UMI 209 UMMISCO, UPMC, IRD, Bondy, France.

\section{Publisher's Note}

Springer Nature remains neutral with regard to jurisdictional claims in published maps and institutional affiliations.

Received: 4 May 2017 Accepted: 14 September 2017 Published online: 03 October 2017

\section{References}

1. Coronel, A, Maulén, C, Pinto, M, Sepúlveda, D: Almost automorphic delayed differential equations and Lasota-Wazewska model. Discrete Contin. Dyn. Syst., Ser. A 37(4), 1959-1977 (2017)

2. Ait Dads, EH, Cieutat, P, Lhachimi, L: Existence of positive almost periodic or ergodic solutions for some neutral nonlinear integral equations. Differ. Integral Equ. 22(11), 1075-1096 (2009)

3. Ding, HS, Chen, YY, N'Guérékata, GM: Existence of positive pseudo almost periodic solutions to a class of neutral integral equations. Nonlinear Anal. 74(18), 7356-7364 (2011)

4. Ortega, R, Tarallo, M: Almost periodic linear differential equations with non-separated solutions. J. Funct. Anal. 237, 402-426 (2006)

5. Pinto, M: Pseudo-almost periodic solutions of neutral integral and differential equations with applications. Nonlinear Anal. 72(12), 4377-4383 (2010)

6. Shen, W, Yi, Y: Almost automorphic and almost periodic dynamics in skew-product semiflows. Mem. Am. Math. Soc 136(647), 1-93 (1998)

7. Torrejón, R: Positive almost periodic solutions of a state-dependent delay nonlinear integral equation. Nonlinear Anal. 20(12), 1383-1416 (1993)

8. Johnson, RA: A linear, almost periodic equation with an almost automorphic solution. Proc. Am. Math. Soc. 82, 199-205 (1981)

9. Bochner, S: A new approach to almost periodicity. Proc. Natl. Acad. Sci. USA 48(12), 2039-2043 (1962)

10. Veech, WA: Almost automorphic functions on groups. Am. J. Math. 87, 719-751 (1965) 
11. Zou, QF, Ding, HS: Almost periodic solutions for a nonlinear integro-differential equation with neutral delay. J. Nonlinear Sci. Appl. 9(6), 4500-4508 (2016)

12. Cooke, KL, Kaplan, JL: A periodicity threshold theorem for epidemics and population growth. Math. Biosci. 31(1-2), 87-104 (1976)

13. Ait Dads, EH, Ezzinbi, K: Existence of positive pseudo almost periodic solution for a class of functional equations arising in epidemic problems. Cybern. Syst. Anal. 30(6), 900-910 (1994)

14. Ait Dads, EH, Ezzinbi, K: Almost periodic solution for some neutral nonlinear integral equation. Nonlinear Anal. 28(9), 1479-1489 (1997)

15. Ait Dads, EH, Ezzinbi, K: Boundedness and almost periodicity for some state-dependent delay differential equations. Electron. J. Differ. Equ. 2002(67), 1 (2002)

16. Fink, A: Almost Periodic Differential Equations. Lecture Notes in Mathematics, vol. 377. Springer, Berlin (1974)

17. Bochner, S: Continuous mappings of almost automorphic and almost periodic functions. Proc. Natl. Acad. Sci. USA 52(4), 907-910 (1964)

18. N'Guérékata, GM: Almost Automorphic and Almost Periodic Functions in Abstract Spaces. Springer, Berlin (2001)

19. Zaidman, S: Almost-Periodic Functions in Abstract Spaces. Pitman Advanced Publishing Program, vol. 126 (1985)

20. Es-sebbar, B: Almost automorphic evolution equations with compact almost automorphic solutions. C. R. Math. 354(11), 1071-1077 (2016)

21. Basit, B, Günzler, H: Spectral criteria for solutions of evolution equations and comments on reduced spectra. (2010). arXiv: 1006.2169

22. Diagana, T: Almost Automorphic Type and Almost Periodic Type Functions in Abstract Spaces. Springer, Berlin (2013)

23. Nicholson, AJ: Compensatory reactions of populations to stresses, and their evolutionary significance. Aust. J. Zool. 2(1), 1-8 (1954)

24. Nicholson, AJ: An outline of the dynamics of animal populations. Aust. J. Zool. 2(1), 9-65 (1954)

25. Gurney, WSC, Blythe, SP, Nisbet, RM: Nicholson's blowflies revisited. Nature 287, 17-21 (1980)

26. Chen, Y: Periodic solutions of delayed periodic Nicholson's blowflies models. Can. Appl. Math. Q. 11(1), $23-28$ (2003)

27. Kolmanovskii, VB, Nosov, VR: Stability of Functional Differential Equations, vol. 180. Academic Press, San Diego (1986)

\section{Submit your manuscript to a SpringerOpen ${ }^{\circ}$ journal and benefit from:}

- Convenient online submission

- Rigorous peer review

- Open access: articles freely available online

- High visibility within the field

- Retaining the copyright to your article

Submit your next manuscript at $\boldsymbol{\nabla}$ springeropen.com 